\title{
COMPUTATIONAL DYNAMICS OF SCARA VARIANT
}

\author{
Manjeet Tummalapalli ${ }^{1}$, Puren Ouyang ${ }^{1 *}$, Wenhui Yue ${ }^{2}$, Luigi Addante ${ }^{1}$ \\ ${ }^{1}$ Department of Aerospace Engineering, Ryerson University, Toronto, Canada \\ ${ }^{2}$ College of Mechanical and Electrical Engineering, Hunan University of Science and Technology, Xiangtan, China \\ *pouyang@ ryerson.ca
}

\begin{abstract}
The SCARA robot has been extensively used for industrial applications for many years. If the applications' requirements need to be modified, per say the physical limitation of the workspace restricts the vertical movement in the system, there may need a modification to the SCARA robot configuration that can accompany to these needed changes. The motivation behind this research is to propose a SCARA variant as an alternative, with the popularity and applications of a SCARA robot. The main objective is to relocate the vertical prismatic joint and study the computational dynamics of the SCARA variant and the performances.

The work done in this research is to design a new SCARA variant of changing the order of joints that limits the vertical motion of the SCARA. The vertical prismatic joint in this modified SCARA variant is changed to axis 2 from axis 3 in the original configuration. The SCARA variant has been analyzed for the forward and inverse kinematics based on the transformation matrix method. The dynamic model for the SCARA variant has been developed by using the Lagrangian equation, and the systematic steps presented in this work can be easily applied for other robotic systems.

The computational torque for each joint of the SCARA variant can be obtained for any prescribed trajectories. In this paper, linear and nonlinear trajectories, such as straight line, ellipse, and circular trajectories have been selected in the simulation study. Consistent results for torque requirements have been observed with the linear trajectory having the least values, followed by ellipse, and the circular trajectory having the highest values. For all the trajectories, the prismatic joint at axis 2 has the highest torque required and the revolute joint at axis 4 has the least. The values of the required forces on the robot are consistent and within the limits of industrial applications. Simulations for required forces show acceptable results. The computational dynamics can be used as a guide tool for the selection of the actuators in the real implementation.

This design can be used as an alternative for the standard SCARA when some new requirements of the application arise. It would be interesting to use the proposed SCARA variant to study the control torques required for trajectories and the tracking errors along the desired trajectory, which can be a future work.
\end{abstract}

